\title{
A Phase I Study to Evaluate the Safety, Tolerability, and Pharmacokinetics of TPNI7IH, a Novel Phosphodiesterase Type 5 Inhibitor, in Healthy Subjects
}

\author{
Hongjie Qian ${ }^{1,2}$ \\ Qian Chen ${ }^{1,2}$ \\ Liyu Liang ${ }^{1,2}$ \\ Yang Zou ${ }^{1,2}$ \\ Huahua $\mathrm{Pu}^{1,2}$ \\ Liang Xin ${ }^{1,2}$ \\ Rong Song ${ }^{1,2}$ \\ Tingting $\mathrm{Li}^{1,2}$ \\ Huijuan Zhu', \\ Yu Wang ${ }^{3}$ \\ Guanghui Tian ${ }^{4}$ \\ Jingshan Shen (iD ${ }^{2,3}$ \\ Hualiang Jiang ${ }^{2,3}$ \\ Chen $\mathrm{Yu}^{\mathrm{I}, 2}$ \\ Zhen Wang iD $^{2,3}$ \\ Jingying Jia 1,2
}

'Central Laboratory, Shanghai Xuhui Central Hospital, Shanghai, 20003I, People's Republic of China; ${ }^{2}$ Shanghai Engineering Research Center of Phase I Clinical Research \& Quality Consistency Evaluation for Drugs, Shanghai, 20003I, People's Republic of China; ${ }^{3}$ CAS Key Laboratory of Receptor Research, Drug Discovery and Design Center, Shanghai Institute of Materia Medica,

Chinese Academy of Sciences, Shanghai, 201203, People's Republic of China; ${ }^{4}$ Vigonvita Life Science Co., Ltd., Suzhou, 215123, People's Republic of China

Correspondence: Jingying Jia

Shanghai Xuhui Central Hospital, No. 966, Huaihai Road(M), Shanghai,

20003I, People's Republic of China

Tel/Fax +86 2l 54030254

Email jyjia@shxh-centerlab.com

Zhen Wang

Shanghai Institute of Materia Medica, Chinese Academy of Sciences, No. 555

Zu Chong Zhi Road, Pudong, Shanghai,

20I203, People's Republic of China

Tel +862 2I 20231000

Fax +86 21 20231962

Email wangzhen@simm.ac.cn
Purpose: TPN171H is a novel, potent and selective phosphodiesterase type 5 (PDE5) inhibitor for the treatment of pulmonary arterial hypertension (PAH). The objective of this study was to evaluate the safety, tolerability, and pharmacokinetics of TPN171H in healthy subjects after single and multiple dosing, in addition, to investigate the food effect on pharmacokinetics and safety of TPN171H.

Methods: The entire study was comprised of three parts: Part I (single ascending-dose study), Part II (food effect study), and Part III (multiple ascending-dose study). A total of 63 healthy subjects were enrolled in the study. TPN171H tablet or placebo was administered per protocol requirements. Blood samples were collected at the designated time points for pharmacokinetic analysis. Safety was assessed by clinical examinations and adverse events. Results: In Part I, AUC and $\mathrm{C}_{\max }$ were proved to be linear within the 5-30 mg dose range. $\mathrm{T}_{1 / 2}$ of TPN171H was $8.02-10.88 \mathrm{~h}$. In Part II, we figured out that TPN171H administration under fed condition could decrease $\mathrm{C}_{\max }$, prolong $\mathrm{T}_{\max }$, but had no effect on AUC. In Part III, the accumulation ratio at steady-state for $\mathrm{AUC}$ and $\mathrm{C}_{\max }$ indicated that $\mathrm{TPN} 171 \mathrm{H}$ has a slight accumulation upon repeated dosing. Subjects were generally tolerable after TPN171H administration. Compared with other PDE5 inhibitors, TPN171H was found to have no impact on blood pressure and color discrimination.

Conclusion: TPN171H was safe and generally tolerated in healthy subjects. Based on the half-life, food effect, and safety profile of TPN171H, we recommend a once-daily, post-meal administration of TPN171H in subsequent clinical studies in healthy subjects and patients with PAH.

Keywords: TPN171H, PDE5 inhibitor, safety, pharmacokinetics, food effect, healthy subjects

\section{Introduction}

Pulmonary arterial hypertension (PAH) is a progressive disease of various origins that is associated with a poor prognosis and results in right heart dysfunction. PAH is now generally regarded as a vasculopathy involving remodeling of the pulmonary vasculature that is characterized by pulmonary vascular cell proliferation and inflammation. ${ }^{1}$ Pulmonary vascular remodeling is considered to be the result of a combination of genetic, epigenetic, and environmental factors, and a variety of vasoactive molecules, ion channels, and signaling pathways exert important regulatory effects. ${ }^{2}$ Currently, the 
treatment algorithm for PAH includes five pharmacological classes: 1) endothelin receptor antagonists, such as bosentan, ambrisentan, and macitentan; 2) phosphodiesterase type 5 (PDE5) inhibitors, such as sildenafil and tadalafil; 3) prostanoids, such as iloprost, epoprostenol, and beraprost; 4) guanidine cyclase stimulators, such as riociguat; 5) prostacyclin receptor agonist, such as selexipag. ${ }^{3-7}$ Although approved drugs are safe and effective, they cannot prevent or reverse the development of the disease. $\mathrm{PAH}$ remains a progressive and fatal disease that seriously threatens the lives of patients. Thus, potential therapeutic targets and new agents to treat PAH are urgently needed. ${ }^{8}$

TPN171H is a novel, potent and selective PDE5 inhibitor for targeted treatment of PAH, like other commercially available PDE5 inhibitors: sildenafil $\left(\right.$ Revatio $^{\circledR}$ ) and tadalafil (Adcirca $^{\circledR}$ ). The nitric oxide (NO) pathway plays a key role in pulmonary vasodilating effects, mediated through its second messenger, cyclic guanosine monophosphate (cGMP). ${ }^{9}$ cGMP is specifically cleaved by phosphodiesterases (PDEs), afterward degrades rapidly. There are eleven subfamilies of human PDEs (PDE1-11), of which PDE5 is the predominant phosphodiesterase isoform in the lung. ${ }^{10} \mathrm{PDE} 5$ is up-regulated in conditions associated with pulmonary hypertension. ${ }^{11}$ By selectively inhibiting PDE5, PDE5 inhibitors can enhance the NO-mediated vasodilatation of the pulmonary artery, and exert anti-proliferative effects on pulmonary artery smooth muscle cells. ${ }^{12}$ In preclinical experiments, TPN171H was proved to have good selectivity, exhibit satisfactory safety and pharmacokinetic profiles in rats and dogs, and exert a longer-lasting effect than sildenafil in animal models. $^{13}$
Therefore, we conducted this study to evaluate the safety, tolerability, and pharmacokinetics of TPN171H in healthy subjects after single and multiple dosing, in addition, to investigate the food effect on pharmacokinetics and safety of TPN171H.

\section{Materials and Methods}

The entire study scheme comprised three parts: Part I, II, and III (Figure 1). Part I and III were randomized, doubleblind, placebo-controlled, single and multiple ascending dose studies. Part II was a randomized, open-label, threecycle, cross-over study to investigate the food effect of TPN171H.

The study was carried out at the Phase I Clinical Research Center of Shanghai Xuhui Central Hospital, Shanghai, China, from December 2017 to September 2018. The study was registered on http://www. chinadrugtrials.org.cn (Registration No.: CTR20171238, CTR20171237, and CTR20181142, respectively). The study protocol was approved by the Ethics Committee of the Shanghai Xuhui Central Hospital and the study was conducted in accordance with the Declaration of Helsinki and Good Clinical Practice. All subjects were required to provide written informed consent before any study-related procedure was performed.

\section{Participants}

Subjects who met the following inclusion criteria, while did not meet the exclusion criteria, were eligible to participate in the study.

Inclusion criteria:

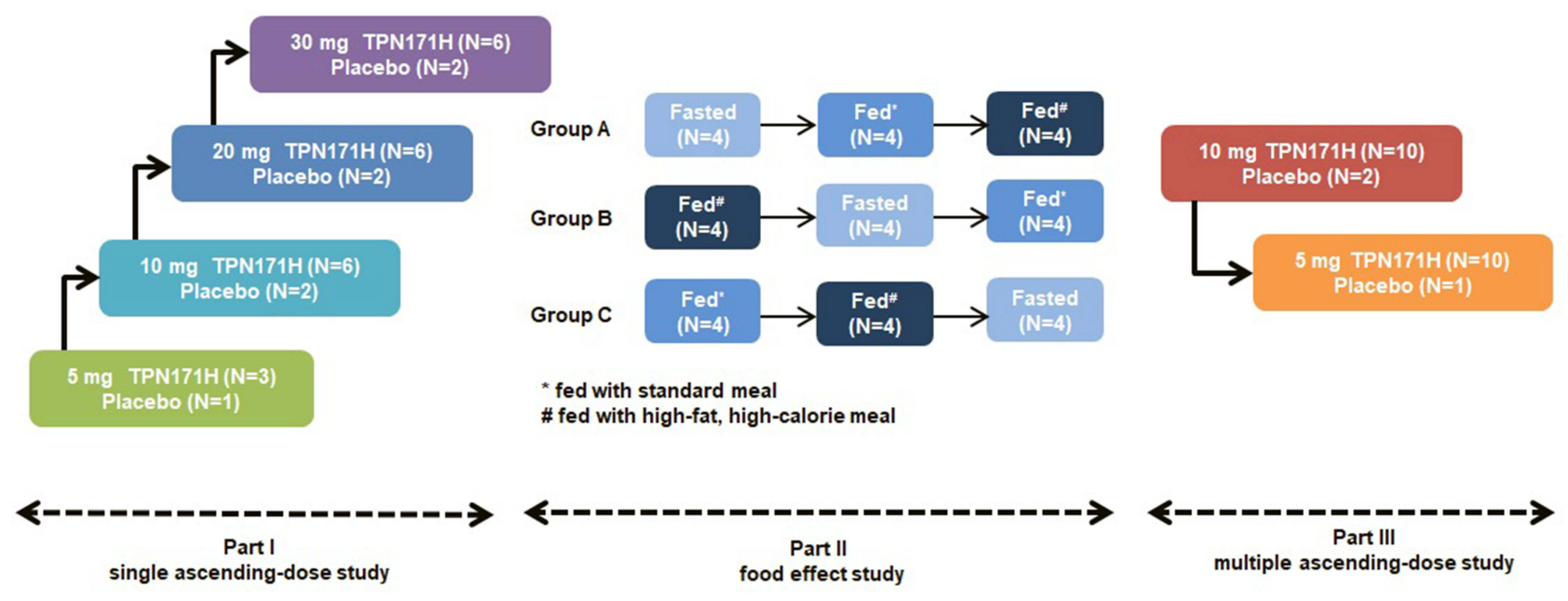

Figure I The scheme of study design. 
- healthy males or females between 18 and 40 years of age;

- bodyweight for males $\geq 50 \mathrm{~kg}$, bodyweight for females $\geq 45 \mathrm{~kg}$, and body mass index (BMI) between 19 and $26 \mathrm{~kg} / \mathrm{m}^{2}$ (included);

- normal vital signs, physical examination, 12-lead ECG and results of laboratory tests, or abnormality with no clinical significance;

- willing to use effective contraceptive measures during the study and within 3 months after the administration of investigational products.

Exclusion criteria:

- allergy history;

- history of any systemic disorders or diseases, history of orthostatic hypotension;

- blurred vision, or color vision defects;

- blood donation or blood loss $\geq 400 \mathrm{~mL}$ within 3 months;

- any concomitant medication within 2 weeks prior to screening;

- participation in any clinical trial within 3 months;

- drug or alcohol addicts, or heavy smokers;

- positive test for hepatitis B, hepatitis C, HIV, or syphilis;

- abnormality with clinical significance in chest X-ray;

- positive pregnancy test for females.

\section{Procedures}

Part I: single ascending-dose study. Based on the nonclinical toxicology data of TPN171H, as well as the dose settings in clinical trials of sildenafil and tadalafil, the estimated maximum recommended starting dose (MRSD) was selected at $5 \mathrm{mg}$, and the estimated maximum dose was selected at $50 \mathrm{mg}$. Six ascending dose groups $(5 \mathrm{mg}$, $10 \mathrm{mg}, 20 \mathrm{mg}, 30 \mathrm{mg}, 40 \mathrm{mg}$, and $50 \mathrm{mg}$ ) were designed in the protocol. The decision to proceed to the next dose level was made by both investigator and sponsor according to the dose-escalation termination criteria defined in the protocol.

Eligible subjects were admitted to the Phase I unit one day before drug administration. On the morning of Day 1, after overnight fasting for $\geq 10 \mathrm{~h}$, subjects were administered TPN171H tablet or placebo at the respective dose level with $240 \mathrm{~mL}$ water under fasted condition. All the subjects stayed at the Phase I unit until $48 \mathrm{~h}$ after dosing with close medical monitoring. A follow-up visit by telephone was performed by investigators on Day 7 .

Part II: food effect study. In this part, eligible subjects were randomized into one of three groups (Group A, B, and C). Each group consisted of three treatment periods (fasted, fed with standard meal, and fed with high-fat, high-calorie meal), and separated by two 3-day washout periods. During the fasted period, a single oral dose of TPN171H tablet $10 \mathrm{mg}$ was administered after a 10-hour overnight fasting. During the two fed periods, a single oral dose of TPN171H tablet $10 \mathrm{mg}$ was administered within 30 minutes after consumption of a standard meal (total calories: approximately $600 \mathrm{kcal}$ ), or a high-fat, highcalorie meal (total calories: approximately $800 \mathrm{kcal}$ $1000 \mathrm{kcal}$, which derives about 150, 250, and 500-600 kcal from protein, carbohydrate, and fat, respectively). All the subjects stayed at the Phase I unit until $48 \mathrm{~h}$ after the last dose. A follow-up visit by telephone was performed by investigators on Day 12 .

Part III: multiple ascending-dose study. Two ascending dose groups (10 mg, $20 \mathrm{mg}$ ) were designed in the protocol originally. Based on the exposure of TPN171H in $10 \mathrm{mg}$ dose group, it was estimated that the $10 \mathrm{mg} /$ day administration would produce the maximum pharmacological effect. Meanwhile, considering the adverse events in the $10 \mathrm{mg}$ group, the investigator and sponsor decided to stop dose ascending and to reduce the dose level to $5 \mathrm{mg}$ instead. Two dose groups (10 mg, $5 \mathrm{mg}$ ) were carried out in the multiple ascending dose study. Eligible subjects were admitted to the Phase I unit one day before drug administration. In the morning of Day 1, Day 3 9, TPN171H tablet or placebo at the respective dose level was administered within 30 minutes after consumption of a standard meal. All the subjects stayed at the Phase I unit until $48 \mathrm{~h}$ after the last dose. A follow-up visit by telephone was performed by investigators on Day 14 .

\section{Safety Assessment}

Safety was assessed by vital signs (blood pressure, heart rate, respiratory rate, and body temperature), physical examinations, Farnsworth-Munsell 100 Hue test, clinical laboratory tests, 12-lead ECGs, and monitoring for adverse events (AEs) throughout the study. AEs were evaluated according to National Cancer Institute Common Terminology Criteria for Adverse Events (CTCAE, version 4.03), and were managed and recorded promptly by qualified investigators according to relevant regulations. All AEs were coded using Medical Dictionary for 
Regulatory Activities (MedDRA, version 20.0). The incidence of AEs was calculated by the number of subjects who experienced at least one AE dividing the total number of subjects.

The dose-escalation termination criteria in single ascending-dose study were determined by AEs. Dose escalation should be terminated when 1) AEs related to study drug of CTCAE Grade 2 and above occur in $\geq 50 \%$ subjects of one dose group; 2) AEs related to study drug of CTCAE Grade 3 and above occur in $\geq 25 \%$ subjects of one dose group; 3) SAE related to study drug occur in at least one subject.

After completing the $30 \mathrm{mg}$ dose group of single ascending-dose study, a preliminary PK analysis was performed, from which $30 \mathrm{mg}$ was found to reach the clinical effective dose level based on the exposure of TPN171H. The investigator and sponsor decided to stop dose escalation, although the dose-escalation termination criteria were not met. Therefore, four ascending dose groups $(5 \mathrm{mg}$, $10 \mathrm{mg}, 20 \mathrm{mg}$, and $30 \mathrm{mg}$ ) were eventually carried out, and the estimated maximum dose $50 \mathrm{mg}$ was not reached.

\section{Biological Sample Collection}

Blood samples (approximately $3 \mathrm{~mL}$ each) were collected from a suitable forearm vein by an indwelling catheter or by immediate venipuncture. In $5 \mathrm{mg}$ group of single ascending-dose study, blood samples were collected at the following 15 time points: 0 (pre-dose), $0.25,0.5,1$, $1.5,2,3,4,6,8,10,12,24,36$, and $48 \mathrm{~h}$ post-dose. Afterwards, the time points were slightly adjusted depending on the pharmacokinetic profile of the $5 \mathrm{mg}$ group. In other dose groups of single ascending-dose study, as well as food effect study, blood samples were collected at the following time points: 0 (pre-dose), 10, 20 minutes, 0.5 , $0.75,1,1.5,2,4,6,8,10,12,24$, and $48 \mathrm{~h}$ post-dose. In multiple ascending-dose study, blood samples were collected at the following time points: 0 (pre-dose), 0.5, 0.75, $1,1.5,2,4,6,8,10,12,24,32$, and 48 h post-dose at Day 1 and Day 9, in addition, 0 hour (pre-dose) at Day 6-8 for determining the steady-state.

The blood samples collected were separated after centrifugation at $4{ }^{\circ} \mathrm{C}, 1500 \mathrm{~g}$ for 10 minutes. The separated plasma was divided into 2 centrifuge tubes (at least $0.5 \mathrm{~mL}$ plasma each), and stored frozen under $-80^{\circ} \mathrm{C}$ until analysis.

\section{Bioanalytical Procedures}

TPN171H is the hydrochloride form of TPN171. In this study, TPN171 in human plasma was analyzed for
TPN171H, determined using a validated LC-MS/MS method. $\mathrm{D}_{3}$-TPN171 was chosen as the internal standard. The calibration curves were linear over the concentration ranges from 0.5 to $500 \mathrm{ng} / \mathrm{mL}$, of which the regression coefficient was $0.9972 \pm 0.0005$. Within-day precisions ranged from 1.8 to $7.3 \%$, and between-day precisions from $2.3 \%$ to $4.9 \%$, accuracies were $95.5-99.8 \%{ }^{14}$

\section{Pharmacokinetic Assessments}

PK parameters were calculated using a non-compartmental model by WinNonlin Software version 7.0 or above (Pharsight, Cary, NC, USA). Main PK parameters included area under the concentration-time curve (AUC), AUC from time zero (pre-dose) to the time of the last measurable concentration $\left(\mathrm{AUC}_{0-\mathrm{t}}\right), \mathrm{AUC}$ from time zero (pre-dose) to infinity $\left(\mathrm{AUC}_{0-\infty}\right)$, maximum observed plasma concentration $\left(\mathrm{C}_{\max }\right)$, time to maximum plasma concentration $\left(T_{\max }\right)$, terminal elimination half-life $\left(t_{1 / 2}\right)$, elimination rate constant $(\mathrm{Ke})$, apparent distribution volume $(\mathrm{Vd} / \mathrm{F})$, clearance rate $(\mathrm{CL} / \mathrm{F})$, and mean retention time (MRT). $\mathrm{AUC}_{0-\mathrm{t}}$ and $\mathrm{AUC}_{0-\infty}$ were calculated using a linear trapezoidal rule method. $\mathrm{T}_{\max }$ and $\mathrm{C}_{\max }$ were based on the actual measured values. In multiple ascending-dose study, degree of fluctuation (DF) and accumulation ratio at steady state $\left(\mathrm{R}_{\mathrm{ac}}\right)$ was also analyzed.

\section{Statistical Analyses}

Statistical analysis was performed using SAS Software version 9.4 or above (SAS Institute, Cary, NC, USA). Descriptive statistics were expressed as arithmetic mean, standard deviation, coefficient of variation, median, maximum, minimum, and geometric mean of each dose group. Frequency and percentage were calculated to summarize categorical variables. $\mathrm{P} \leq 0.05$ was considered to be statistically significant.

In single ascending-dose study, the dose linear relationship was assessed using confidence interval criteria. First, linear regression of PK parameters and doses was carried out. The regression equation was expressed as $\ln (\mathrm{PK})=\alpha$ $+\beta \times \ln$ (Dose) after logarithmic transformation of PK parameters and doses. Then, the $90 \%$ confidence interval of the estimated slope $(\beta)$ was calculated. PK parameters was determined to be linearly relative to dose, in the condition that the $90 \%$ confidence interval of $\beta$ for $\mathrm{AUC}_{0-\mathrm{t}}$ and $\mathrm{AUC}_{0-\infty}$ was within the acceptance interval $80-125 \%$, while that for $\mathrm{C}_{\max }$ was within $70-143 \%$.

In the food effect study, $\mathrm{PK}$ parameters $\mathrm{AUC}_{0-\mathrm{t}}, \mathrm{AUC}_{0-\infty}$, and $\mathrm{C}_{\max }$ under different dietary conditions were statistically 
analyzed after logarithmic transformation. The antilogarithmic transformation was performed for these results, to obtain the least-squares geometric mean, point estimation of the mean ratio, and estimated $90 \%$ confidence interval of the ratio. The least-squares mean and the difference between each two treatment groups (high-fat meal vs fasted, standard meal vs fasted, high-fat meal vs standard meal) were calculated for each PK parameter, respectively. Food intake was considered to have no effect on the bioavailability of TPN171H, in the condition that the $90 \%$ confidence interval of the geometric mean ratio of $\mathrm{AUC}_{0-\mathrm{t}}, \mathrm{AUC}_{0-\infty}$ and $\mathrm{C}_{\max }$ under fed condition was within $80-125 \%$ of that under fasted condition. ${ }^{15}$

\section{Results}

\section{Demographic Profile}

A total of 63 eligible subjects were enrolled in the study, including 28 in Part I, 12 in Part II, and 23 in Part III, respectively. Subject distribution is displayed in Figure 1. Expect for 1 subject in Part III who discontinued from the study due to personal reason, other 62 subjects completed the study as per protocol. All 63 exposed subjects were included in the safety analysis set and the full analysis set. The demographic profile of all enrolled subjects is demonstrated in Table 1.

\section{Pharmacokinetic Properties Part I: Single Ascending-Dose Study}

The main PK parameters in each dose group after a single dose of TPN171H are summarized in Table 2, and the mean plasma drug concentration-time curves are shown in Figure 2.

The PK characteristics of TPN171H indicated by the parameters of $T_{\max }, t_{1 / 2}, V d / F$, and $C L / F$ were similar in the four dose groups $(\mathrm{P}>0.05)$, while AUC parameters and $\mathrm{C}_{\max }$ were dose-dependent. $\mathrm{AUC}_{0-\mathrm{t}}$ was $275.97 \pm 35.32$, $541.77 \pm 157.78, \quad 1069.09 \pm 161.03$ and $1656.44 \pm 345.38$ $\mathrm{h} \cdot \mathrm{ng} / \mathrm{mL}$ for $5 \mathrm{mg}, 10 \mathrm{mg}, 20 \mathrm{mg}$, and $30 \mathrm{mg}$ dose groups, respectively. $\mathrm{AUC}_{0-\infty}$ was $287.00 \pm 42.67,560.45 \pm 160.17$, $1089.64 \pm 158.37$ and $1709.27 \pm 376.39 \mathrm{~h} \cdot \mathrm{ng} / \mathrm{mL}$ for the four dose groups. $\mathrm{C}_{\max }$ was $40.4 \pm 8.62,80.1 \pm 23.48,145.8$ \pm 30.05 , and $236.9 \pm 104.07 \mathrm{ng} / \mathrm{mL}$ for the four dose groups. Confidence interval criteria were used to assess the dose linear relationship. The $90 \%$ confidence interval of the estimated slope for $\mathrm{C}_{\max }, \mathrm{AUC}_{0-\mathrm{t}}$, and $\mathrm{AUC}_{0-\infty}$ was $76.30-112.80 \%, \quad 87.40-112.60 \%$ and $86.50-112.00 \%$ respectively, which was contained completely within the acceptance intervals, indicating that AUC parameters and

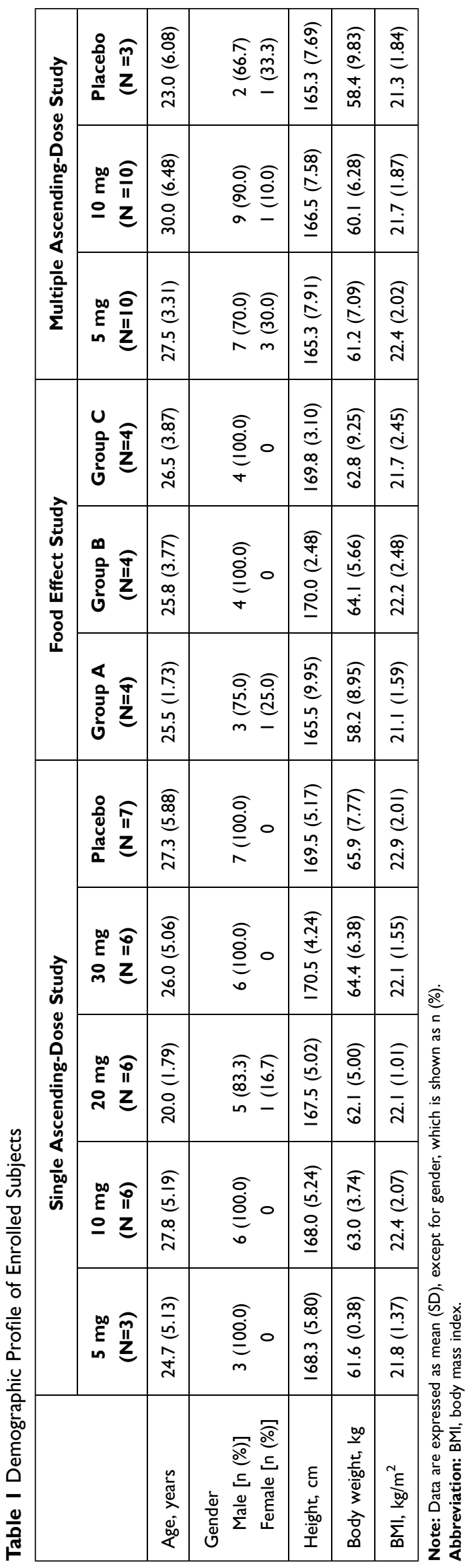


Table 2 The Main PK Parameters in Each Dose Group After a Single Dose of TPNI7IH

\begin{tabular}{|c|c|c|c|c|}
\hline Pharmacokinetic Parameters & 5 mg $(\mathbf{N}=3)$ & $10 \mathrm{mg}(\mathrm{N}=6)$ & $20 \mathrm{mg}(\mathrm{N}=6)$ & $30 \mathrm{mg}(\mathrm{N}=6)$ \\
\hline $\mathrm{AUC}_{0-\mathrm{t}}(\mathrm{h} \cdot \mathrm{ng} / \mathrm{mL})^{* *}$ & $275.97(35.32)$ & $541.77(157.78)$ & $1069.09(161.03)$ & I $656.44(345.38)$ \\
\hline$A \cup C_{0-\infty}(h \cdot n g / m L) * *$ & $287.00(42.67)$ & $560.45(160.17)$ & $1089.64(158.37)$ & I709.27 (376.39) \\
\hline $\mathrm{C}_{\max }(\mathrm{ng} / \mathrm{mL})^{* *}$ & $40.4(8.62)$ & $80.1(23.48)$ & $145.8(30.05)$ & $236.9(104.07)$ \\
\hline $\mathrm{T}_{\max }(\mathrm{h})$ & $0.5(0.5,0.5)$ & $0.6(0.3,2.0)$ & $1.3(1.0,2.0)$ & $0.9(0.5,4.0)$ \\
\hline $\mathrm{t}_{1 / 2}(\mathrm{~h})$ & $10.88(4.58)$ & $8.02(1.69)$ & $8.05(1.55)$ & $9.93(1.40)$ \\
\hline $\operatorname{Ke}(\mathrm{I} / \mathrm{h})$ & $0.07(0.03)$ & $0.09(0.02)$ & $0.09(0.02)$ & $0.07(0.01)$ \\
\hline $\mathrm{Vd} / \mathrm{F}(\mathrm{L})$ & $268.29(80.19)$ & $217.14(67.35)$ & $213.09(27.75)$ & $255.83(24.92)$ \\
\hline $\mathrm{CL} / \mathrm{F}(\mathrm{L} / \mathrm{h})$ & $17.66(24.48)$ & $19.02(49.97)$ & $18.734(3|.4|)$ & $18.35(43.87)$ \\
\hline MRT (h) & $10.93(2.67)$ & $9.50(1.66)$ & $8.97(1.80)$ & $11.08(2.18)$ \\
\hline
\end{tabular}

Notes: Data are expressed as mean (SD), except for $\mathrm{T}_{\max }$, which is shown as median (min, max). **P<0.0I: The difference of the PK parameter among different dose groups is considered to be statistically significant.

Abbreviations: $\mathrm{AUC}_{0-\mathrm{t}}$, area under the concentration-time curve from time zero to the time of the last measurable concentration; $A \cup \mathrm{C}_{0-\infty}$, area under the concentration-time curve from time zero to infinity; $C_{\max }$, maximum observed plasma concentration; $T_{\max }$, time to maximum plasma concentration; $t_{1 / 2}$, terminal elimination half-life; Ke, elimination rate constant; Vd/F, apparent distribution volume; CL/F, clearance rate; MRT, mean retention time.

$\mathrm{C}_{\max }$ were linearly relative to dose within 5-30 $\mathrm{mg}$ dose range. The dose linearity plots are demonstrated in Figure $3 . \mathrm{T}_{1 / 2}$ of $\mathrm{TPN} 171 \mathrm{H}$ was within $8.02-10.88 \mathrm{~h}$ as observed.

\section{Part II: Food Effect Study}

The key PK parameters of TPN171H under fasted and fed conditions after a single oral dose of $10 \mathrm{mg}$ TPN171H are listed in Table 3, and the mean plasma drug concentrationtime curves under fasted and fed conditions are displayed in Figure 4.

The ratios of the geometric mean of $\mathrm{AUC}_{0-\mathrm{t}}, \mathrm{AUC}_{0-\infty}$ and $\mathrm{C}_{\max }$ for $\mathrm{TPN} 171 \mathrm{H}$ under the fed condition with a standard meal to those observed under fasted condition (90\% confidence interval) were $97.79 \% \quad(93.87 \%$ $\sim 101.89 \%), \quad 98.34 \% \quad(94.35 \% \sim 102.50 \%)$ and $77.29 \%$ $(65.88 \% \sim 90.68 \%)$, respectively. The ratios of the geometric mean of $\mathrm{AUC}_{0-\mathrm{t}}, \mathrm{AUC}_{0-\infty}$ and $\mathrm{C}_{\max }$ for TPN171H under the fed condition with a high-fat, highcalorie meal to those observed under fasted condition (90\% confidence interval) were $104.63 \% \quad(100.42 \%$ $\sim 109.00 \%), \quad 104.99 \%$ (100.73\% 109.43\%) and $91.09 \%$ (77.64\% 106.87\%), respectively. The ratio of the geometric mean of $\mathrm{C}_{\max }$ exceeded the equivalent range $80-$ $125 \%$, however, that of AUC parameters were within the range. The median $\mathrm{T}_{\max }$ of $\mathrm{TPN} 171 \mathrm{H}$ under fasted condition, fed condition with a standard meal, fed condition with a high-fat, high-calorie meal was $1.5 \mathrm{~h}, 4.0 \mathrm{~h}$, and $2.0 \mathrm{~h} \quad(\mathrm{P}<0.05)$. From above, we figured out that TPN171H administration under fed condition could decrease the peak concentration of absorption, while prolonging the time to the peak, as compared with the fasted condition. The differences were more evident in subjects fed with a standard meal.

\section{Part III: Multiple Ascending-Dose Study}

The main PK parameters of TPN171H in multiple-dose study at D1, D9 are presented in Table 4. The mean plasma drug concentration-time curves at D1, D9 of $5 \mathrm{mg}$, and $10 \mathrm{mg}$ dose groups are demonstrated in Figure 5.

Plasma concentration of TPN171H at D6-D9 pre-dose indicated that steady-state was achieved at D7 after fiveday repeated dosing for $5 \mathrm{mg}$ dose group, while at D6 after four-day repeated dosing for $10 \mathrm{mg}$ dose group $(\mathrm{P}>0.05)$.

For the $5 \mathrm{mg}$ dose group, the mean $\mathrm{t}_{1 / 2}$ after the last dosing was longer than that after the first dosing (11.20 $\mathrm{h}$ vs $8.41 \mathrm{~h}, \mathrm{P}<0.01$ ), and for the $10 \mathrm{mg}$ dose group, the mean MRT at D9 was longer than that at D1 (14.44 h vs $12.25 \mathrm{~h}, \mathrm{P}<0.01$ ), suggesting that continuous dosing of TPN171H could prolong the elimination rate in the human body. As for $\mathrm{AUC}_{0-\mathrm{t}}, \mathrm{AUC}_{0-\infty}$, and $\mathrm{C}_{\max }$, the drug exposure increased after repeated dosing. The accumulation ratio at steady-state $\left(\mathrm{R}_{\mathrm{ac}}\right)$ for $\mathrm{AUC}_{0-\mathrm{t}}$ was $141.19 \%$, $137.84 \%$ for $5 \mathrm{mg}$ and $10 \mathrm{mg}$ dose groups, and $\mathrm{R}_{\mathrm{ac}}$ for $\mathrm{C}_{\max }$ was $121.48 \%, 109.66 \%$ for these two dose groups, which indicated that TPN171H has a slight accumulation upon repeated dosing.

\section{Safety}

\section{Part l: Single Ascending-Dose Study}

AEs occurred in every dose group except for the $5 \mathrm{mg}$ dose group. The number (incidence) of subjects experiencing AEs for $10 \mathrm{mg}, 20 \mathrm{mg}, 30 \mathrm{mg}$ dose group and placebo group was $3(50 \%), 6(100 \%), 5(83.3 \%)$ and $3(42.9 \%)$, 


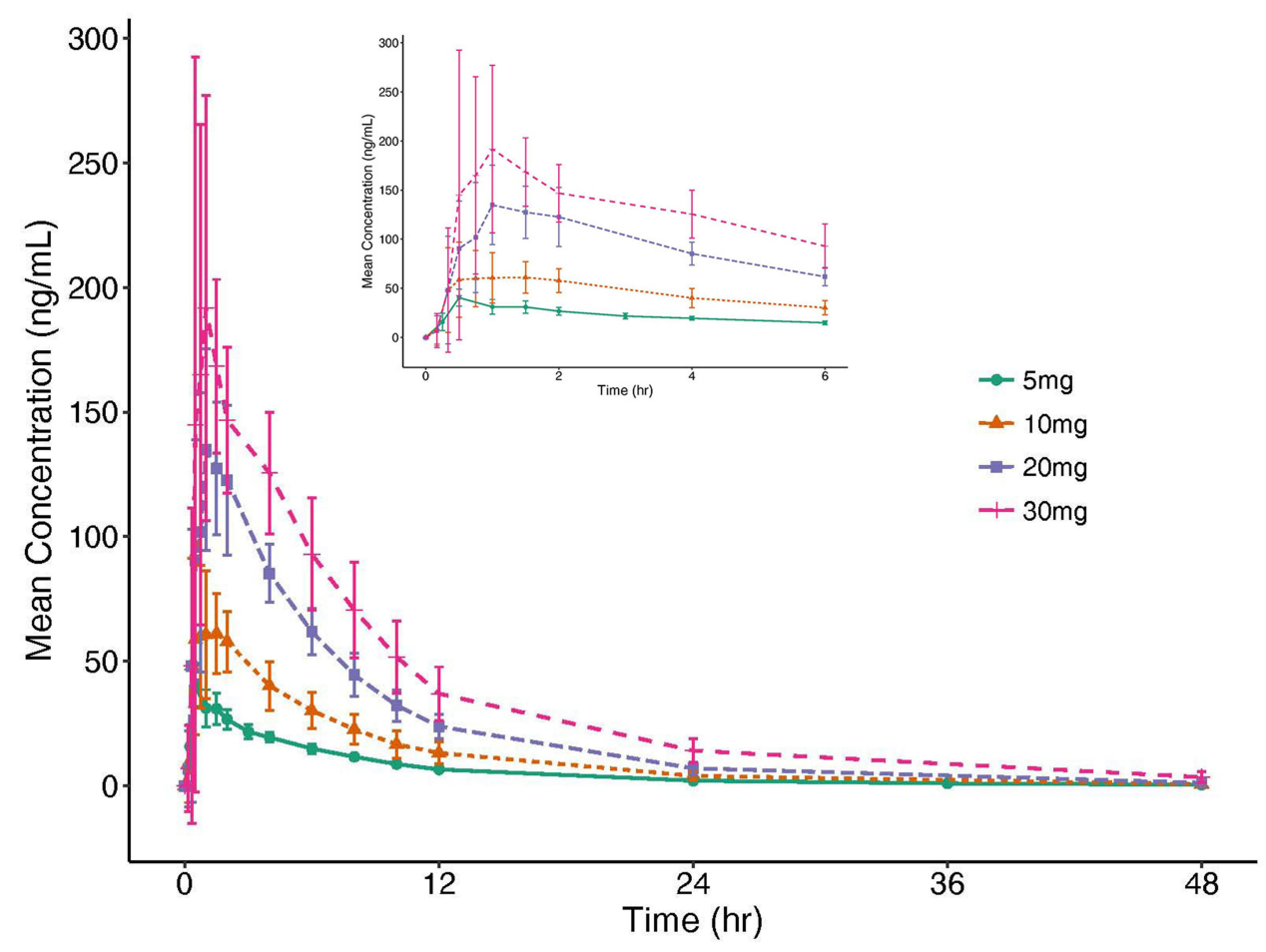

Figure 2 The mean plasma drug concentration-time curves in each dose group after a single dose of TPNI7IH.
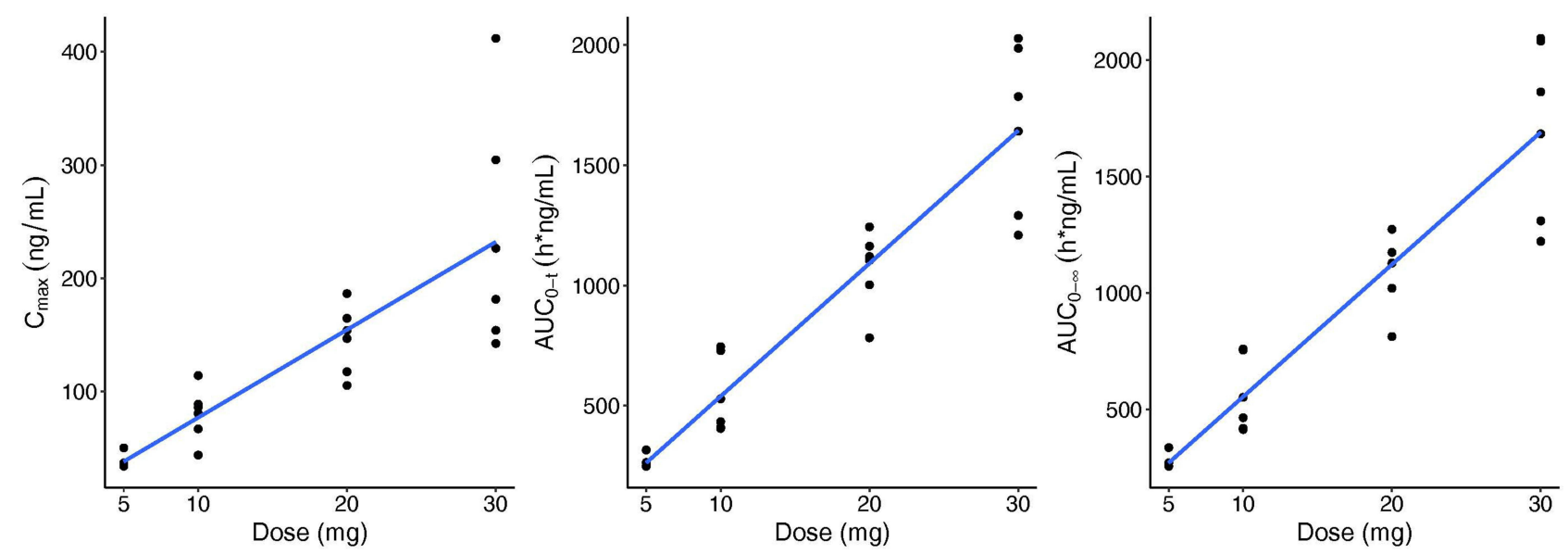

Figure 3 Dose linearity plots of $C_{\max }$ and AUC parameters in single ascending-dose study.

respectively. Among the 34 cases reported AEs after TPN171H administration, 33 cases were deemed to be related to study drug with CTCAE Grade 1, which did not meet the dose-escalation termination criteria defined in the protocol. The most common drug-related AEs were dizziness, spontaneous telotism, headache, flushing, nasal obstruction, and hiccup. No serious adverse event occurred throughout the study, and no subject withdrew from the study due to AE.

\section{Part II: Food Effect Study}

Twenty-two cases AEs were happened in 8 subjects of 12 enrolled subjects, which were all CTCAE Grade 1 in severity, and deemed to be related to study drug. The number (incidence) of subjects experiencing AEs under fasted condition, fed condition with a standard meal, fed condition with a high-fat, high-calorie meal was 5 (41.7\%), 5 $(41.7 \%)$, and $4(33.3 \%)$, suggesting food might have little influence on the safety profile of TPN171H. Just like the 
Table 3 The Main PK Parameters of TPNI7IH Under Fasted and Fed Conditions After a Single Oral Dose of 10 Mg TPNI7IH

\begin{tabular}{|c|c|c|c|}
\hline Pharmacokinetic Parameters & Fasted $(\mathrm{N}=\mid 2)$ & $\begin{array}{l}\text { Fed with Standard Meal } \\
\qquad(N=12)\end{array}$ & $\begin{array}{l}\text { Fed with High-Fat, High-Calorie Meal } \\
\qquad(N=\mid 2)\end{array}$ \\
\hline $\mathrm{AUC}_{0-\mathrm{t}}(\mathrm{h} \cdot \mathrm{ng} / \mathrm{mL})^{*}$ & $635.62(84.93)$ & $622.00(84.90)$ & $662.03(59.03)$ \\
\hline$A \cup C_{0-\infty}(h \cdot n g / m L) *$ & $648.87(85.24)$ & $638.43(85.55)$ & $678.58(63.24)$ \\
\hline $\mathrm{C}_{\max }(\mathrm{ng} / \mathrm{mL})^{*}$ & $74.44(13.50)$ & $57.67(12.42)$ & $69.42(21.29)$ \\
\hline$T_{\max }(h)^{*}$ & $1.50(0.50,2.00)$ & $4.00(0.75,4.00)$ & $2.00(0.33,6.00)$ \\
\hline$t_{1 / 2}(h)$ & $9.04(0.72)$ & $9.30(1.17)$ & $9.15(0.96)$ \\
\hline $\mathrm{Vd} / \mathrm{F}(\mathrm{L})$ & $205.45(40.36)$ & $215.42(51.996)$ & $195.72(25.31)$ \\
\hline $\mathrm{CL} / \mathrm{F}(\mathrm{L} / \mathrm{h})^{*}$ & $15.66(2.11)$ & $15.9(2.25)$ & $14.86(1.42)$ \\
\hline
\end{tabular}

Notes: Data are expressed as mean (SD), except for $\mathrm{T}_{\max }$, which is shown as median (min, max). ${ }^{*} \mathrm{P}<0.05$ : The difference of the $\mathrm{PK}$ parameter under fasted and fed conditions is considered to be statistically significant.

Abbreviations: $\mathrm{AUC}_{0-\mathrm{t}}$, area under the concentration-time curve from time zero to the time of the last measurable concentration; $A \cup C_{0-\infty}$, area under the concentration-time curve from time zero to infinity; $C_{\max }$, maximum observed plasma concentration; $T_{\max }$, time to maximum plasma concentration; $t_{1 / 2}$, terminal elimination half-life; $\mathrm{Vd} / \mathrm{F}$, apparent distribution volume; $\mathrm{CL} / \mathrm{F}$, clearance rate.

single ascending-dose study, the most common drug-related AEs in the food effect study were nasal obstruction, head discomfort, flushing, and headache. No difference in $\mathrm{AE}$ type was discovered for fasted and fed state. No serious adverse event was reported, and no subject withdrew from the study due to AE.

\section{Part III: Multiple Ascending-Dose Study}

In $10 \mathrm{mg}$ dose group, 212 cases AEs occurred in all the 10 subjects $(100 \%)$ administered TPN171H, of which 211 cases were judged to be related to the study drug. The most common drug-related AEs were spontaneous telotism, dizziness, headache, head discomfort, nasal obstruction, palpitation, flushing, and nausea. Among these AEs, 1 rhinorrhea, 1 cough, 1 drug rash, 1 ophthalmalgia, 1 itchy eye, and 1 gum pain were reported as Grade 2 in severity. Although the majority of AEs were CTCAE Grade 1, the investigator and sponsor decided to stop the dose ascending to $20 \mathrm{mg}$ for subjects' sake, instead to reduce the dose level to $5 \mathrm{mg}$.

In the $5 \mathrm{mg}$ dose group, 148 cases AEs occurred, including 141 cases related to the study drug. The

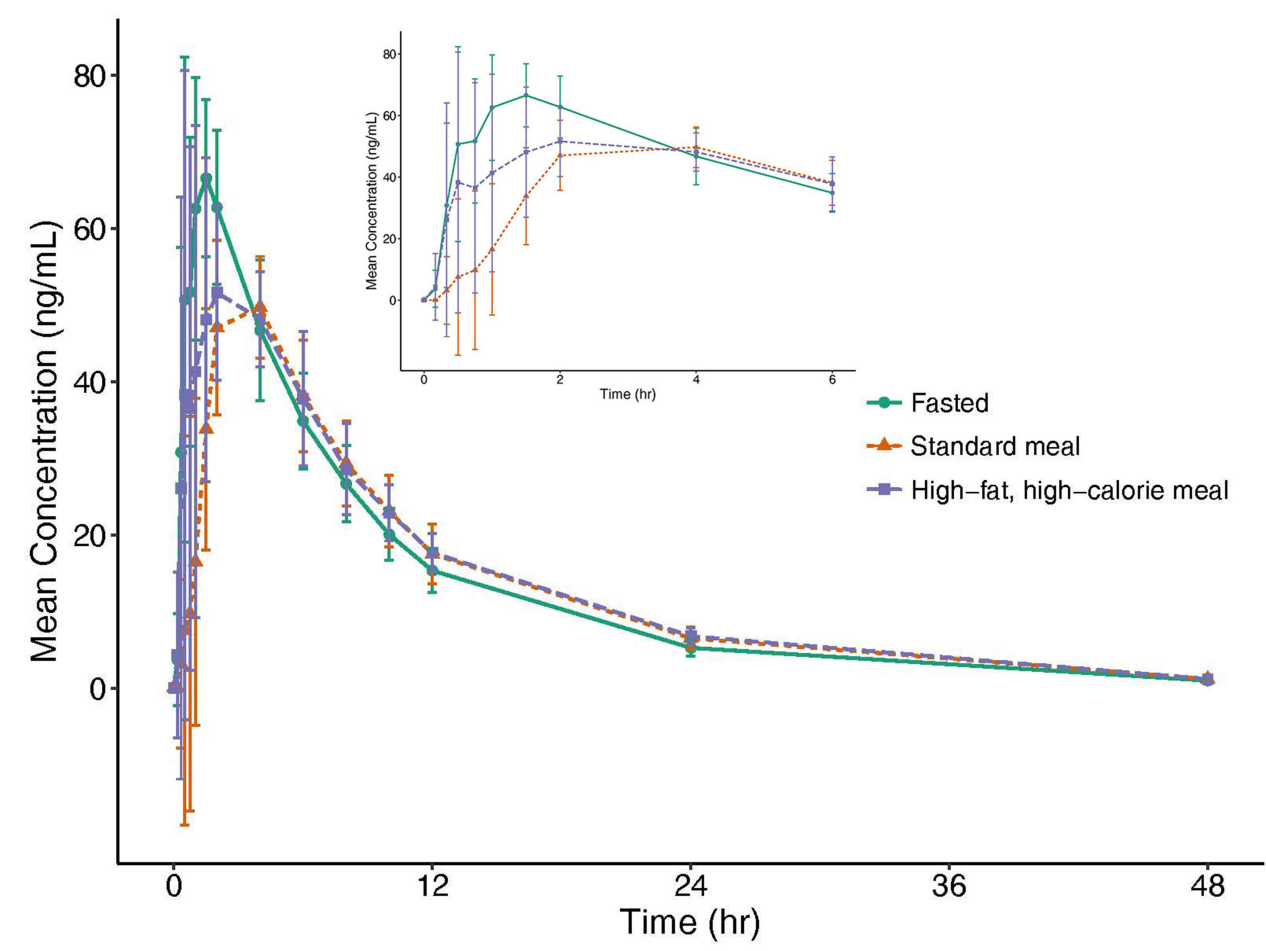

Figure 4 The mean plasma drug concentration-time curves under fasted and fed conditions. 
Table 4 The Main PK Parameters of TPNI7IH in Multiple Ascending-Dose Study at DI, D9

\begin{tabular}{|c|c|c|c|c|}
\hline \multirow[t]{2}{*}{ Pharmacokinetic Parameters } & \multicolumn{2}{|c|}{$5 \mathrm{mg}(\mathrm{N}=10)$} & \multicolumn{2}{|c|}{$10 \mathrm{mg}(\mathrm{N}=10)$} \\
\hline & DI & D9 & DI & D9 \\
\hline$A \cup C_{0-t}(h \cdot n g / m L)$ & 259.44 (38.56) & $367.30(74.25)^{* *}$ & $615.32(117.04)$ & $838.54(152.23)^{* *}$ \\
\hline $\mathrm{AUC}_{0-\infty}(\mathrm{h} \cdot \mathrm{ng} / \mathrm{mL})$ & $272.16(41.36)$ & $388.05(83.03)^{* *}$ & $634.84(121.01)$ & $877.34(165.74)^{* *}$ \\
\hline $\mathrm{C}_{\max }(\mathrm{ng} / \mathrm{mL})$ & $26.09(6.61)$ & $30.84(6.34)^{*}$ & $62.43(15.26)$ & $66.49(13.21)$ \\
\hline $\mathrm{T}_{\max }(\mathrm{h})$ & $3.00(0.50,4.02)$ & $2.00(0.50,4.00)$ & $2.00(1.00,4.00)$ & $4.00(1.00,6.00)$ \\
\hline$t_{1 / 2}(h)$ & $8.4 I(1.31)$ & II $.20(3.83)^{* *}$ & $10.3 \mid(2.66)$ & $10.96(1.43)$ \\
\hline $\mathrm{CL} / \mathrm{F}(\mathrm{L} / \mathrm{h})$ & I8.84 (3.52) & $16.28(2.98)^{* *}$ & $16.30(3.32)$ & $14.59(2.62)^{*}$ \\
\hline MRT (h) & II.25 (2.0I) & I 3.25 (3.29)* & $12.25(1.79)$ & $14.44(2.09)^{* *}$ \\
\hline DF (\%) & - & $207.28(35.08)$ & - & 198.65 (32.35) \\
\hline $\mathrm{R}_{\mathrm{ac}}\left(\mathrm{C}_{\max }\right)(\%)$ & - & $121.48(22.26)$ & - & $109.66(20.15)$ \\
\hline $\mathrm{R}_{\mathrm{ac}}\left(A \cup \mathrm{C}_{0-\mathrm{t}}\right)(\%)$ & - & 141.19 (16.76) & - & I37.84 (20.82) \\
\hline
\end{tabular}

Notes: Data are expressed as mean (SD), except for $T_{\max }$, which is shown as median (min, max). $* P<0.05$, **P $<0.01$ : The difference of the PK parameter between $\mathrm{DI}$ and D9 is considered to be statistically significant.

Abbreviations: $\mathrm{AUC}_{0-\mathrm{t}}$, area under the concentration-time curve from time zero to the time of the last measurable concentration; $A \cup \mathrm{C}_{0-\infty}$, area under the concentration-time curve from time zero to infinity; $C_{\max }$, maximum observed plasma concentration; $T_{\max }$, time to maximum plasma concentration; $t_{1 / 2}$, terminal elimination half-life; CL/F, clearance rate; MRT, mean retention time; DF, degree of fluctuation; $\mathrm{R}_{\mathrm{ac}}$, accumulation ratio at steady state.

incidence of $\mathrm{AE}$ was $100 \%$ (10 in 10). Similar to the $10 \mathrm{mg}$ group, the most common drug-related AEs were spontaneous telotism, headache, head discomfort, swelling of the eye, myalgia, nasal obstruction, dizziness, flushing, and blurred vision, among which 3 headache, 2 chest discomfort, 2 palpitation, and 1 toothache were reported as Grade 2.

Only 1 of 3 subjects (33.3\%) in the placebo group experienced 2 cases AEs. The incidence was remarkably lower than that of the TPN171H group.

\section{Other Safety Assessments}

There were no clinically significant changes in vital signs, especially in blood pressure. Throughout the entire study, no increase was observed for the scores of Farnsworth-Munsell 100 Hue test post-dose compared with baseline, indicating TPN171H does not affect subjects' color discrimination.

\section{Discussion}

TPN171H is a novel, potent and selective inhibitor of PDE5 for potential use in PAH, the same as other commercially available PDE5 inhibitors: sildenafil and tadalafil. In preclinical experiments, TPN171H was proved to have good selectivity, exhibit satisfactory safety and pharmacokinetic profiles in rats and dogs, and exert a longerlasting effect than sildenafil in animal models. ${ }^{13}$ This study was designed to evaluate the safety, tolerability, and pharmacokinetics of TPN171H in healthy subjects.

In Table 5, we summarized data drawn from this study as well as reported in literatures, to compare the PK characteristics of TPN171H in humans with other marketed PDE5 inhibitors: sildenafil and tadalafil. ${ }^{16}$ TPN171H demonstrated satisfactory dose linearity within the 5-30 mg dose range for AUC parameters and $\mathrm{C}_{\max }$. We could conclude from this table that TPN171H had similar $\mathrm{T}_{\max }$ as sildenafil and tadalafil, however had moderate absorption, distribution, and elimination behavior between sildenafil and tadalafil. Due to the short half-life (3-5 h) of sildenafil, it's required to take sildenafil three times daily for treatment of $\mathrm{PAH}$, which is not convenient for PAH patients. ${ }^{5}$ But for TPN171H, the half-life was $8.02-10.88 \mathrm{~h}$, which could be administered once daily for treatment of PAH.

In the food effect study, we observed that high-fat, high-calorie meal slightly reduced the peak concentration of TPN $171 \mathrm{H}$ by $9 \%$, and prolonged the time to peak a little bit by $0.5 \mathrm{~h}$, compared with the fasted condition. The above-mentioned findings were even more obvious for a standard meal, which reduced the peak concentration by about $23 \%$, and remarkably prolonged the time to peak by $2.5 \mathrm{~h}$. Nevertheless, fed with a standard meal and a high-fat, high-calorie meal did not influence systemic exposure and safety profile of TPN171H. The food effect of TPN171H was quite similar to sildenafil. As reported in the literature, administration of sildenafil after a high-fat meal caused reductions in the rate of absorption and extent of systemic exposure. The time-to-peak $\left(\mathrm{T}_{\max }\right)$ was delayed by approximately $1 \mathrm{~h}$, and maximum concentration $\left(\mathrm{C}_{\max }\right)$ was reduced by $29 \%$. However, the systemic exposure of sildenafil after a high-fat meal was reduced by 


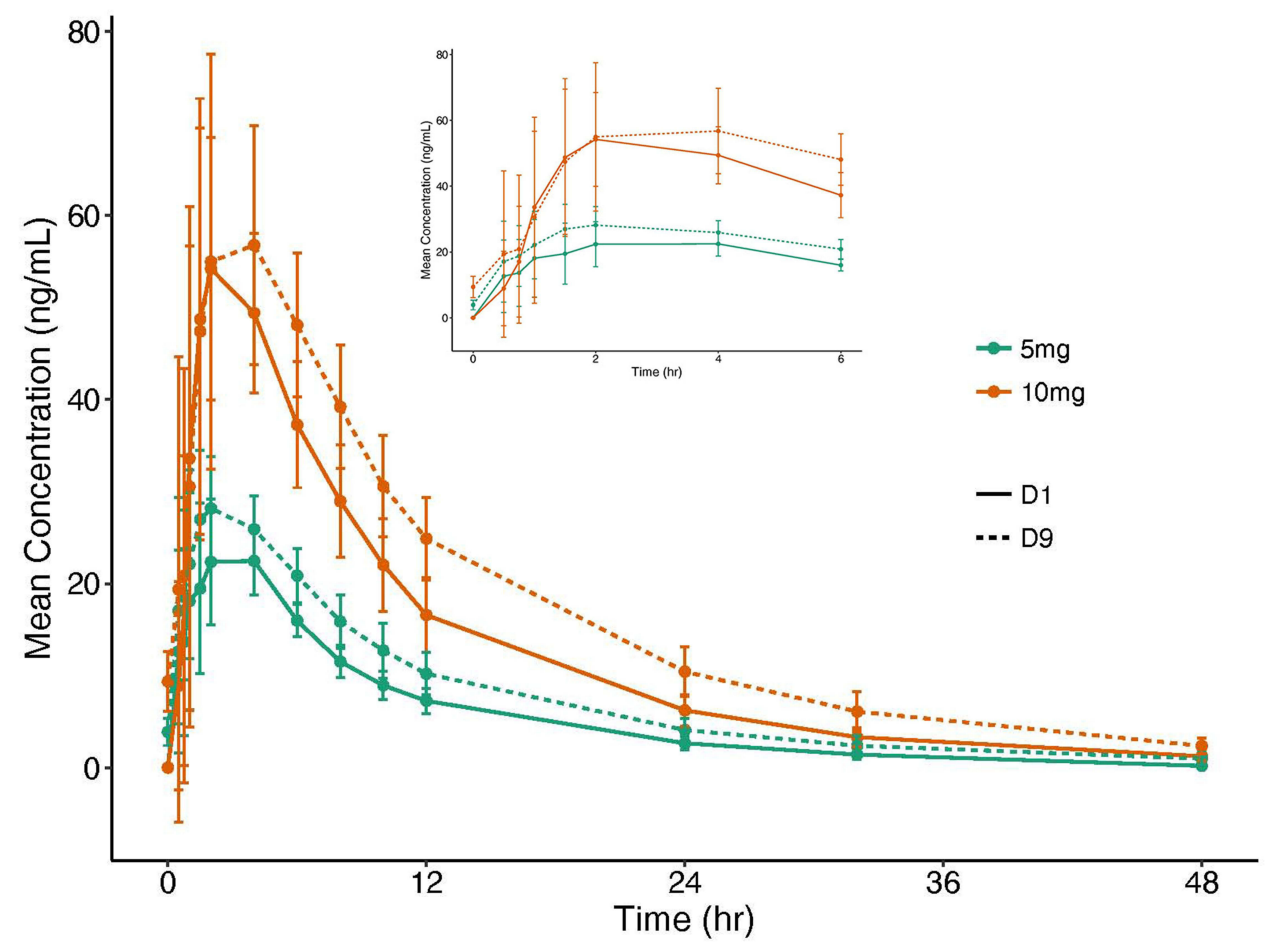

Figure 5 The mean plasma drug concentration-time curves at DI, D9 of $5 \mathrm{mg}$ and $10 \mathrm{mg}$ dose groups.

$11 \%$, which was different from TPN171H. ${ }^{17}$ For tadalafil, the PK properties were not affected by food. ${ }^{18}$

Based on the result of the food effect study, we recommend a post-meal administration of TPN171H in subsequent clinical studies in healthy subjects and patients with
$\mathrm{PAH}$, to reduce adverse drug reactions (ADR) around $\mathrm{T}_{\max }$.

In multiple ascending-dose study, TPN171H tablet or placebo was administered after consumption of a standard meal. Thus, the PK profile at D1 in multiple ascending-dose

Table 5 Comparative Clinical Pharmacokinetics of PDE5 Inhibitors Including TPNI7IH. ${ }^{16}$

\begin{tabular}{|c|c|c|c|}
\hline & TPNI7IH & Sildenafil & Tadalafil \\
\hline $\begin{array}{l}\text { Available doses } \\
(\mathrm{mg})\end{array}$ & $5,10,20,30$ & $25,50,100$ & $5,10,20$ \\
\hline$A \cup C_{0-t}(h \cdot n g / m L)$ & $1069.09(20 \mathrm{mg})$ & $1685(100 \mathrm{mg})$ & $8066(20 \mathrm{mg})$ \\
\hline $\mathrm{C}_{\max }(\mathrm{ng} / \mathrm{mL})$ & I 45.8 (20 mg) & $560(100 \mathrm{mg})$ & 378 (20 mg) \\
\hline $\mathrm{T}_{\max }(\mathrm{h})$ & $0.5-1.3$ & I $(0.5-2)$ & $2(0.5-6)$ \\
\hline $\mathrm{t}_{1 / 2}(\mathrm{~h})$ & $8.02-10.88$ & $3-5$ & 17.5 \\
\hline $\mathrm{Vd} / \mathrm{F}(\mathrm{L})$ & $213.09(20 \mathrm{mg})$ & $>310$ & $60-70$ \\
\hline $\mathrm{CL} / \mathrm{F}(\mathrm{L} / \mathrm{h})$ & $18(20 \mathrm{mg})$ & $4 \mathrm{I}$ (CL, $50 \mathrm{mg}$, intravenous) & 2.5 (20 mg) \\
\hline Dose linearity & Linear & Slightly nonlinear & Linear \\
\hline Food effect & $\mathrm{T}_{\max } 2.5 \mathrm{~h} \uparrow, \mathrm{C}_{\max } 23 \% \downarrow, \mathrm{AUC} \rightarrow$ (standard & 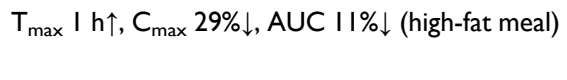 & $\begin{array}{c}\text { No significant } \\
\text { effect }\end{array}$ \\
\hline
\end{tabular}

Abbreviations: $\mathrm{AUC}_{0-\mathrm{t}}$, area under the concentration-time curve from time zero to the time of the last measurable concentration; $\mathrm{C}_{\text {max }}$, maximum observed plasma concentration; $\mathrm{T}_{\max }$, time to maximum plasma concentration; $\mathrm{t}_{\mathrm{I} / 2}$, terminal elimination half-life; $\mathrm{Vd} / \mathrm{F}$, apparent distribution volume; $\mathrm{CL} / \mathrm{F}$, clearance rate. 
study was different from that at the corresponding dose level in single ascending-dose study, in which TPN171H tablet or placebo was administered under fasted condition. Consistent with the result of the food effect study, prolonged $\mathrm{T}_{\max }$ and decreased $\mathrm{C}_{\max }$ was observed in multipledose study.

After 7-day continuous dosing of TPN171H, $\mathrm{R}_{\mathrm{ac}}$ for $\mathrm{AUC}_{0-\mathrm{t}}$ was $141.19 \%, 137.84 \%$, and for $\mathrm{C}_{\max }$ was $121.48 \%, 109.66 \%$ for $5 \mathrm{mg}$ and $10 \mathrm{mg}$ dose groups, respectively, indicating that $\mathrm{TPN} 171 \mathrm{H}$ has a slight accumulation upon repeated dosing. The incidence of $\mathrm{AE}$ was $100 \%$ for subjects who received TPN171H, which was higher than that in the placebo group. As expected, the most common drug-related AEs were spontaneous telotism, dizziness, headache, head discomfort, nasal obstruction, palpitation, flushing, and nausea. Considering the PK and safety characteristics in multiple-dose study, we propose a once-daily dosing regimen for further clinical studies in PAH patients. Besides, an even lower starting dosage at $2.5 \mathrm{mg}$ was suggested in patients.

TPN171H is a selective PDE5 inhibitor with better potency and selectivity than sildenafil and tadalafil. ${ }^{13}$ As is known to all, there are eleven sub-families of human PDEs (PDE1-11), possessing a conserved C-terminal catalytic domain for hydrolysis of the ubiquitous second messenger, cAMP, and cGMP. High selectivity is thus crucial to a PDE5 inhibitor, and inhibition of other PDE isozymes rather than PDE5 may lead to off-target adverse effects. For example, PDE1 is expressed in the brain, myocardial cells, vascular smooth muscle cells, therefore, non-selectivity of PDE5 inhibitors concerning all PDE1 subtypes may induce vasodilatation, flushing, and tachycardia. PDE6 is expressed in the retina, thus inhibition of this enzyme can cause visual disturbances. PDE11 is expressed in the liver, kidney, pituitary gland, skeletal muscle, myocardium, prostate, corpus cavernosum, and to a high degree in the testes, inhibition of PDE11 can cause abnormal spermatogenesis in mice, while may induce myalgia and back pain in clinical use. ${ }^{19}$ Sildenafil and tadalafil reported several adverse reactions due to nonselectivity of PDE5, such as headache, flushing, dyspepsia, abnormal vision, nasal congestion, back pain, myalgia, nausea, nasopharyngitis, upper respiratory tract infection, and dizziness. The observed drug-related AEs in our study were basically consistent with those reported for sildenafil and tadalafil. In patients prescribed sildenafil at single oral doses of $100 \mathrm{mg}$ and $200 \mathrm{mg}$, transient dose-related impairment of color discrimination was detected using the Farnsworth-Munsell 100-hue test, which was reported as difficulties in discriminating blue/green. ${ }^{20}$ In our study, however, no impact on color discrimination was detected for TPN171H at any dosage using the FarnsworthMunsell 100 Hue test, which proved that TPN171H has good selectivity over PDE6. Besides, decrease of blood pressure (max decreasing amplitude: $8.3 / 5.3 \mathrm{mmHg}$ ) was observed in healthy subjects following administration of sildenafil $100 \mathrm{mg},{ }^{20}$ while TPN171 $\mathrm{H}$ was found to have no influence on blood pressure in our study.

Despite the incidence and variety of the drug-related AEs, subjects were generally tolerable after TPN171H administration. No subject withdrew from the study due to AEs. ADRs were drug concentration-related, which means most ADRs could mitigate spontaneously with metabolism and elimination of TPN171H in the human body. The most common drug-related AE observed in this study was spontaneous telotism that occurred among male subjects, and the reaction could relieve without intervention. In light of the adverse reaction on the penis, TPN171H can be a potential candidate for the treatment of erectile dysfunction (ED) in addition to PAH.

Considering that the pharmacokinetic characteristics in patients may be different from that in healthy subjects, we suggest a phase $\mathrm{Ib}$ study could be performed in PAH patients, to investigate the safety, tolerability, and pharmacokinetics of TPN171H in patients, meanwhile, to explore the efficacy in patients preliminarily. As discussed above, we propose a once-daily dosing regimen, as well as a lower starting dosage at $2.5 \mathrm{mg}$ of TPN171H for the phase Ib study.

\section{Conclusions}

TPN171H was safe and generally tolerated in healthy subjects. Based on the half-life, food effect, and safety profile of TPN171H, we recommend a once-daily, postmeal administration of TPN171H in subsequent clinical studies in healthy subjects and patients with PAH.

\section{Data Sharing Statement}

The authors intend to share individual deidentified participant data, such as the individual PK and the safety data, in form of supplementary data. The data will be available from the corresponding authors Jingying Jia and Zhen Wang.

\section{Ethical Statement}

The study protocol was approved by the Ethics Committee of the Shanghai Xuhui Central Hospital and the study was 
conducted in accordance with the Declaration of Helsinki and Good Clinical Practice. All subjects were required to provide written informed consent before any study-related procedure was performed.

\section{Acknowledgments}

The authors sincerely thank the healthy subjects and staffs who participated in the clinical study. The authors acknowledge Clinical Service Center Co., Ltd for clinical monitoring of this study. The authors also acknowledge Meta Clinical Technology Co., Ltd for statistical analysis of this study.

\section{Funding}

This study was sponsored by Vigonvita Life Science Co., Ltd. The study was also supported by the National Science \& Technology Major Project "Key New Drug Creation and Manufacturing Program”, China (No. 2018ZX09711002), and the Strategic Priority Research Program of Chinese Academy of Sciences (No. XDA12040103).

\section{Disclosure}

Zhen Wang, Yu Wang, Jingshan Shen and Hualiang Jiang are the employees of Shanghai Institute of Materia Medica, the sponsor of this study. Guanghui Tian is the employee of Vigonvita Life Science Co., Ltd, the cosponsor of this study.

Professor Jingshan Shen reports grants from National Health Commission of the People's Republic of China, grants from Chinese Academy of Sciences, during the conduct of the study; holds the stock of Vigonvita Life Science Co., Ltd., outside the submitted work. In addition, Professor Jingshan Shen has a patent PCT/CN2009/ 001418 issued to Shanghai Institute of Materia Medica, Chinese Academy of Sciences, a patent PCT/CN2018/ 093965 issued to Shanghai Institute of Materia Medica, Chinese Academy of Sciences.

Professor Hualiang Jiang reports grants from National Health Commission of the People's Republic of China, grants from Chinese Academy of Sciences, during the conduct of the study. In addition, Professor Hualiang Jiang has a patent PCT/CN2018/093965 issued, a patent PCT/CN2009/001418 issued.

Professor Zhen Wang reports grants from National Health Commission of the People's Republic of China, grants from Chinese Academy of Sciences, during the conduct of the study. In addition, Professor Zhen Wang has a patent PCT/CN2009/001418 issued, a patent PCT/ CN2018/093965 issued.

The other authors report no conflicts of interest related to this work.

\section{References}

1. Schermuly RT, Ghofrani HA, Wilkins MR, Grimminger F. Mechanisms of disease: pulmonary arterial hypertension. Nat Rev Cardiol. 2011;8:443-455. doi:10.1038/nrcardio.2011.87

2. Humbert M, Guignabert C, Bonnet S, et al. Pathology and pathobiology of pulmonary hypertension: state of the art and research perspectives. Eur Respir J. 2019;53:1801887. doi:10.1183/ 13993003.01887-2018

3. Galiè N, Corris PA, Frost A, et al. Updated treatment algorithm of pulmonary arterial hypertension. $J$ Am Coll Cardiol. 2013;62(25): D60-D72. doi:10.1016/j.jacc.2013.10.031

4. Galiè N, Ghofrani HA, Torbicki A, et al. Sildenafil citrate therapy for pulmonary arterial hypertension. $N$ Engl $J$ Med. 2005;353:2148-2157. doi:10.1056/NEJMoa050010

5. Galiè N, Brundage BH, Ghofrani HA, et al. Tadalafil therapy for pulmonary arterial hypertension. Circulation. 2009;119:2894-2903. doi:10.1161/CIRCULATIONAHA.108.839274

6. Pulido T, Adzerikho I, Channick RN, et al. Macitentan and morbidity and mortality in pulmonary arterial hypertension. $N$ Engl $J$ Med. 2013;369:809-818. doi:10.1056/NEJMoa1213917

7. Makowski CT, Rissmiller RW, Bullington WM. Riociguat: a novel new drug for treatment of pulmonary hypertension. Pharmacotherapy. 2015;35(5):502-519. doi:10.1002/phar.1592

8. Zheng W, Wang Z, Jiang XR, Zhao QJ, Shen JS. Targeted drugs for treatment of pulmonary arterial hypertension: past, present, and future perspectives. $J$ Med Chem. 2020;63(24):15153-15186. doi:10.1021/acs.jmedchem.0c01093

9. Moncada S, Higgs A. The L-arginine-nitric oxide pathway. $N$ Engl J Med. 1993;329(27):2002-2012. doi:10.1056/ NEJM199312303292706

10. Rabe KF, Tenor H, Dent G, Schudt C, Nakashima M, Magnussen H. Identification of PDE isozymes in human pulmonary artery and effect of selective PDE inhibitors. Am J Physiol. 1994;266:L536-L543. doi:10.1152/ajplung.1994.266.5.L536

11. Jernigan NL, Resta TC. Chronic hypoxia attenuates cGMP-dependent pulmonary vasodilation. Am J Physiol Lung Cell Mol Physiol. 2002;282:L1366-L1375. doi:10.1152/ajplung.00273.2001

12. Wharton J, Strange JW, Moller GMO, et al. Antiproliferative effects of phosphodiesterase type 5 inhibition in human pulmonary artery cells. Am J Respir Crit Care Med. 2005;172(1):105-113. doi:10.1164/rccm.200411-1587OC

13. Wang Z, Jiang XR, Zhang XL, et al. Pharmacokinetic-driven optimization of $4(3 \mathrm{H})$-pyrimidinones as phosphodiesterase type 5 inhibitors leading to TPN171, a clinical candidate for the treatment of pulmonary arterial hypertension. $J$ Med Chem. 2019;62(10):4979-4990. doi:10.1021/acs.jmedchem.9b00123

14. Zhang MQ, Liu HM, He JJ, et al. Liquid chromatography-tandem mass spectrometric assay for TPN171 in human plasma. $J$ Pharm Biomed Anal. 2020;191:113634. doi:10.1016/j.jpba.2020. 113634

15. Guidance for Industry: Food-Effect Bioavailability and Fed Bioequivalence Studies. U.S. Department of Health and Human Services, Food and Drug Administration, Center for Drug Evaluation and Research (CDER); 2002. Available from: https:// www.fda.gov/regulatory-information/search-fda-guidance-docu ments/food-effect-bioavailability-and-fed-bioequivalence-studies. Accessed July 02, 2021. 
16. Gupta M, Kovar A, Meibohm B. The clinical pharmacokinetics of phosphodiesterase-5 inhibitors for erectile dysfunction. J Clin Pharmacol. 2005;45:987-1003. doi:10.1177/0091270005 276847

17. Nichols DJ, Muirhead GJ, Harness JA. Pharmacokinetics of sildenafil after single oral doses in healthy male subjects: absolute bioavailability, food effects and dose proportionality. Br J Clin Pharmacol. 2002;53(Suppl1):5S-12S. doi:10.1046/j.0306-5251.2001.00027.x
18. Brock GB. Tadalafil: a new agent for erectile dysfunction. Can J Urol. 2003;10(Suppl 1):17-22.

19. Bischoff E. Potency, selectivity, and consequences of nonselectivity of PDE inhibition. Int J Impot Res. 2004;16:S11-S14. doi:10.1038/sj. ijir.3901208

20. VIAGRA ${ }^{\circledR}$ (sildenafil citrate) tablets [prescribing information]. U.S.; December, 2017.

\section{Publish your work in this journal}

Drug Design, Development and Therapy is an international, peerreviewed open-access journal that spans the spectrum of drug design and development through to clinical applications. Clinical outcomes, patient safety, and programs for the development and effective, safe, and sustained use of medicines are a feature of the journal, which has also been accepted for indexing on PubMed Central. The manuscript management system is completely online and includes a very quick and fair peer-review system, which is all easy to use. Visit http://www. dovepress.com/testimonials.php to read real quotes from published authors.

Submit your manuscript here: https://www.dovepress.com/drug-design-development-and-therapy-journal 